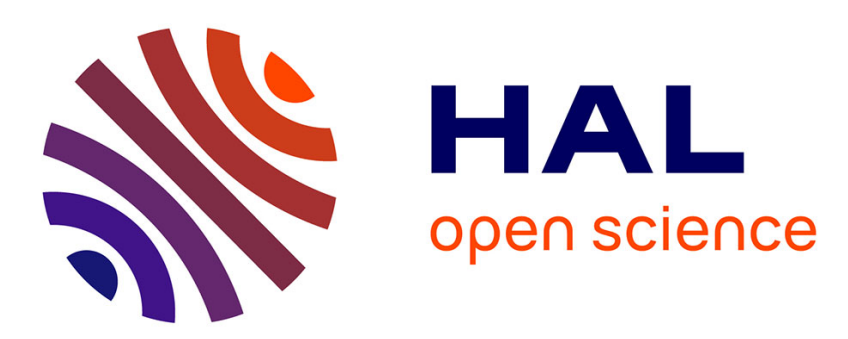

\title{
Experimental observation of near-heteroclinic cycles in the von Karman swirling flow
}

\author{
C Nore, F Moisy, L. Quartier
}

\section{To cite this version:}

C Nore, F Moisy, L. Quartier. Experimental observation of near-heteroclinic cycles in the von Karman swirling flow. Physics of Fluids, 2005, 17 (6), pp.064103. 10.1063/1.1926827 . hal-01591092

\section{HAL Id: hal-01591092 \\ https://hal.science/hal-01591092}

Submitted on 20 Sep 2017

HAL is a multi-disciplinary open access archive for the deposit and dissemination of scientific research documents, whether they are published or not. The documents may come from teaching and research institutions in France or abroad, or from public or private research centers.
L'archive ouverte pluridisciplinaire HAL, est destinée au dépôt et à la diffusion de documents scientifiques de niveau recherche, publiés ou non, émanant des établissements d'enseignement et de recherche français ou étrangers, des laboratoires publics ou privés. 


\title{
Experimental observation of near-heteroclinic cycles in the von Kármán swirling flow
}

\author{
C. Nore \\ Département de Physique, Université Paris XI, 91405 Orsay Cedex, France and Laboratoire d'Informatique \\ pour la Mécanique et les Sciences de l'Ingénieur, CNRS, Boîte Postale 133, \\ 91403 Orsay Cedex, France \\ F. Moisy ${ }^{\mathrm{a})}$ \\ Fluides, Automatique et Systèmes Thermiques, Bâtiment 502, Campus Universitaire, \\ 91405 Orsay Cedex, France \\ L. Quartier \\ Laboratoire de Physique Statistique, Ecole Normale Supérieure, 24 rue Lhomond, \\ 75231 Paris Cedex 05, France
}

(Received 15 September 2004; accepted 12 April 2005; published online 27 May 2005)

\begin{abstract}
The bifurcations and the nonlinear dynamics of the von Kármán swirling flow between exactly counterrotating disks in a stationary cylinder are experimentally investigated by means of visualizations and particle image velocimetry. A regime diagram of the different flow states is determined as a function of the height-to-radius ratio $\Gamma$ and the Reynolds number Re based on disks rotation speed and cylinder radius. Among the steady and time-dependent states found in the experiment, robust near-heteroclinic cycles, which link two unstable states of azimuthal wavenumber $m=2$, are observed and characterized in detail for $\Gamma=2$. These are compared with the numerical findings of Nore et al. ["The 1:2 mode interaction in exactly counter-rotating von Kármán swirling flow," J. Fluid Mech 477, 51 (2003)], with a particular emphasis on the influence of the imperfection and the noise of the experimental setup. (C) 2005 American Institute of Physics.

[DOI: $10.1063 / 1.1926827$ ]
\end{abstract}

\section{INTRODUCTION}

A characteristic feature of dynamical systems in the presence of symmetries is the possible existence of robust heteroclinic cycles. ${ }^{1-4}$ A heteroclinic cycle connects a set of saddle points in a certain range of parameters, and the time spent in the neighborhood of each saddle point increases indefinitely as the cycle is approached. This phenomenon has gained increasing attention after the work of Busse and Heikes ${ }^{5}$ on a dynamical model describing the behavior of the Küppers-Lortz instability of rotating convection rolls. ${ }^{6}$ In a certain range of control parameters, a system of rolls is unstable to rolls at an angle of about $60^{\circ}$. This behavior can be described by a set of three coupled nonlinear real ordinary equations, ${ }^{5}$ which was first proposed as a model for population biology. ${ }^{7}$ Nonrotating convection also provides physical situations where the competition between two modes may create heteroclinic cycles. Indeed a normal form involving four real amplitude equations was derived for convection in two superimposed fluid layers, heated from below and separated by a conducting plate. ${ }^{8}$ This normal form was exhaustively analyzed by Proctor and Jones ${ }^{9}$ and Armbruster et al. ${ }^{10}$ as the $1: 2$ mode resonance in the presence of $O(2)$ symmetry. The ubiquitous occurrence of $m: n$ mode resonances has been revealed in two-dimensional Rayleigh-Bénard convection without Boussinesq symmetry, ${ }^{11,12}$ in turbulent boundary

$\overline{\left.{ }^{a}\right) \text { Electronic mail: moisy@ fast.u-psud.fr }}$ layers, ${ }^{13,14}$ and in the Kuramoto-Sivashinsky equation ${ }^{15}$ using dynamical system theory.

In contrast, experimental observations of heteroclinic cycles are less common. Hu et al. ${ }^{16}$ have investigated multiple instabilities of rotating layers of fluid heated from below, including the already mentioned Küppers-Lortz instability. Its experimental signature is the propagation of fronts where rolls of one orientation grow to replace rolls in the unstable orientation. An experiment in a porous plug burner at low pressure shows ordered patterns of cellular flame consisting of concentric rings of cells. ${ }^{17,18}$ In certain regions of parameter space, these rings appear intermittently, persisting for various periods of time and abruptly changing to the same or different number of cells.

The flow in cylindrical geometries produced by rotating disks with internal shear layers provides another example where heteroclinic cycles may be found, as first shown by Nore et al. ${ }^{19}$ in numerical computations. This flow configuration has recently been the subject of considerable interest, as it provides a simple hydrodynamical system where the symmetries play a key role in the sequence of bifurcations. ${ }^{19-25}$ In the particular case of exactly counterrotating disks with a stationary sidewall at a height-to-radius ratio $\Gamma=2$, when the disk rotation rate is increased, the axisymmetric basic state becomes unstable through a transition which resembles the Kelvin-Helmholtz instability of the equatorial azimuthal free shear layer created by the counterrotation of the top and bottom disks. This instability gives rise to steady states with one or two corotating radial vortices 
generated by the saturation of the corresponding azimuthal mode. Accompanying this instability are more complex dynamics such as traveling waves, modulated traveling waves, and heteroclinic cycles. These cycles connect two states, each containing two corotating vortices and related by $\pi / 2$ rotation about the cylinder axis.

The critical Reynolds numbers for this flow have been investigated when the aspect ratio is varied between 0.5 and 3 and the counterrotation of the two disks maintained. ${ }^{25}$ The $1: 2$ codimension-two point at which the $m=1$ and the $m$ $=2$ thresholds coincide has been located at $\Gamma(1: 2)=1.64$. Heteroclinic cycles may occur in a large region of parameter space when the $m=1$ threshold precedes that of the $m=2$, i.e., when $\Gamma>\Gamma(1: 2)$, but only extremely near the codimension-two point when the order is reversed [i.e., when $\Gamma<\Gamma(1: 2)]$. At $\Gamma=2$, only periodic cycles with heteroclinic type behavior have been numerically observed and are thus called near-heteroclinic cycles. ${ }^{19}$ As was first pointed out by Busse and Heikes, ${ }^{5}$ numerical noise prevents the flow from staying an indefinite amount of time in a neighborhood of a saddle state, leading to a periodic cycle.

The main purpose of this paper is to experimentally investigate the sequence of states which occur when the Reynolds number is varied for aspect ratios between 0.5 and 2.5, and to characterize in detail the near-heteroclinic cycles observed at $\Gamma=2$. The outline of the paper is as follows: Sec. II describes the experimental setup and visualization and measurement systems. In Sec. III, the regime diagram and the steady states obtained for different aspect ratios are reported. Section IV describes the symmetries of the flow, and numerical results for the case $\Gamma=2$ are summarized. Section $V$ is devoted to the study of the steady and unsteady flow states for $\Gamma=2$, with a particular emphasis on the near-heteroclinic cycles. Section VI contains our conclusion.

\section{EXPERIMENTAL SETUP}

\section{A. Experimental cell}

The experimental cell is sketched in Fig. 1. The cylindrical container, made of Plexiglas, of inner radius $R_{c}=35.5$ and $120 \mathrm{~mm}$ long, is mounted vertically. The working fluid is silicon oil of kinematic viscosity $\nu=10^{-4} \mathrm{~m}^{2} \mathrm{~s}^{-1}$ at $25^{\circ} \mathrm{C}$. The top and bottom disks, of radius $R_{d}=34.5 \mathrm{~mm}$, are immersed into the fluid that totally fills the cylinder. The distance between the disks, $H$, can be adjusted between a few millimeters up to $89 \mathrm{~mm}$. In the present paper, the aspect ratio, defined as $\Gamma=H / R_{c}$, is varied between 0.5 and 2.5. Finally, the cylinder is immersed into a rectangular water tank, in order to minimize optical distortion.

The disks are driven by a dc motor, and constrained to rotate with exactly opposite velocities. The angular velocity $\Omega$ can be set between 10 and $64 \mathrm{rad} / \mathrm{s}$ with a stability of $0.1 \%$. Typical runs are of $10^{4}$ rotations, and the waiting time between runs is at least 2000 rotations in order to avoid transient effects. The temperature, measured in the outer water bath, showed a maximum drift of $\pm 0.1{ }^{\circ} \mathrm{C}$ during a run, leading to an uncertainty of $\pm 0.3 \%$ for the viscosity. The Reynolds number, based on the cylinder radius $R_{c}$ and the disk velocity $\Omega R_{d}$,

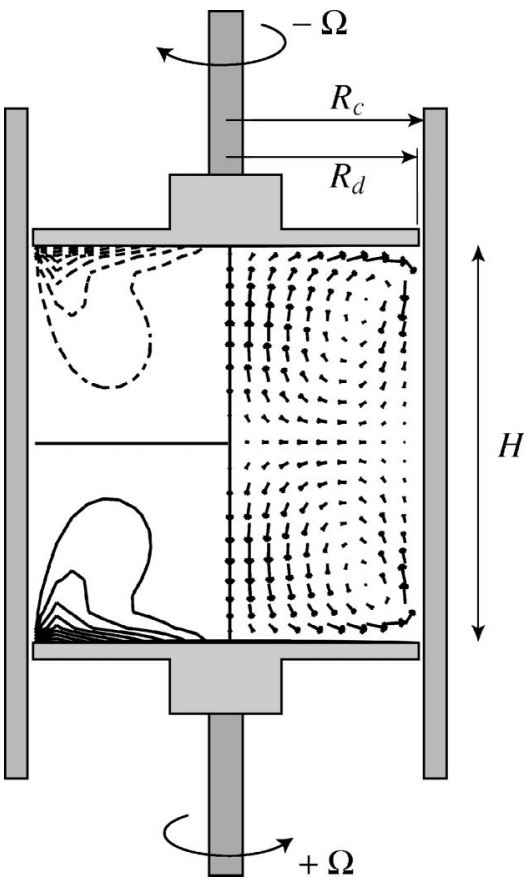

FIG. 1. Experimental cell. The inset illustrates azimuthal velocity contours (left) and the meridional velocity field (right) of the axisymmetric flow determined by numerical simulation (Ref. 19).

$$
\operatorname{Re}=\Omega R_{d} R_{c} / \nu,
$$

is varied between 140 and 600 . Note that, in the numerical simulations of Nore et al., ${ }^{19,25}$ the two radii are equal and denoted by $R$, so that the two control parameters are simply given by $\Gamma=H / R$ and $\operatorname{Re}=\Omega R^{2} / \nu$.

The basic axisymmetric steady flow is also depicted in Fig. 1. It consists of a predominant equatorial shear layer produced by the counterrotating top and bottom disks, and separating two regions with opposite senses of azimuthal velocities (left part of Fig. 1). Two second-order recirculation zones due to Ekman pumping in the neighborhood of each disk (right part of Fig. 1) converge at midheight and form an inward radial jet.

\section{B. Flow visualization}

Visualizations using Iriodin flakes were performed in order to determine the various flow states which occur as the control parameters $(\Gamma, \mathrm{Re})$ are varied. Although crude, this visualization method is convenient as a first approach, as it allows us to easily define the onset modes and the associated critical Reynolds numbers.

Two mirrors, placed at angles of $120^{\circ}$ on each side of the cylinder, allow for visualization around the whole perimeter. The pictures, taken from a charge-coupled device (CCD) camera, were processed in order to unfold the three views: the front view is shown in the left picture, while the side views were reversed and rescaled, and are shown in the middle and right pictures (see Figs. 2 and 3).

Along the equatorial shear layer, the vertical velocity vanishes and the Iriodin flakes are essentially horizontal. Therefore the shear layer appears as a dark horizontal line when seen by reflection and as a bright line when seen by 
(a)
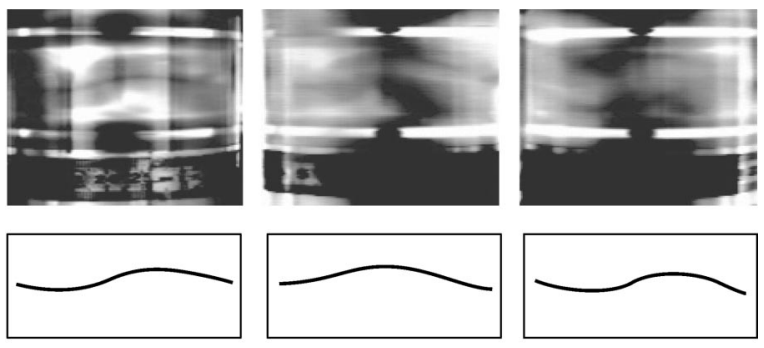

(b)
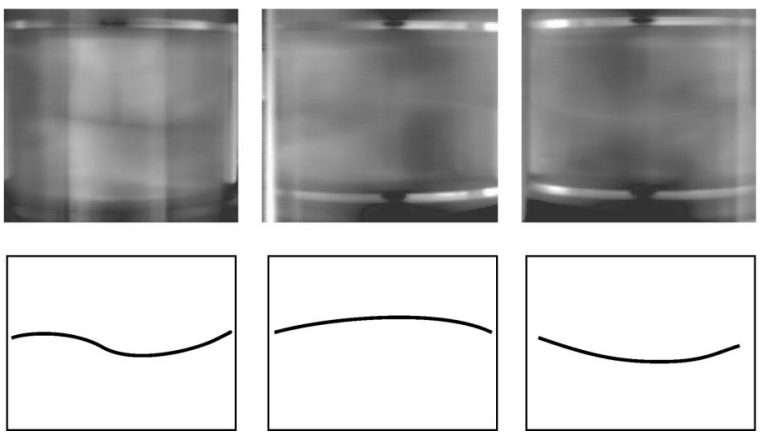

FIG. 2. Azimuthal modulation of the shear layer, as observed using Iriodin flakes, for various aspect ratios $\Gamma$. The pictures were processed in order to unfold the three views: the front view is shown in the left picture, while the side views are reversed and rescaled, and are shown in the middle and right pictures. The shear layer is seen by reflection in the left picture (dark line) and by transmission in the two other pictures (bright line). The mode corresponds to the number of extrema along the unfolded perimeter. The lines drawn below the pictures are guides for the eye. (a) $\Gamma=0.75, m_{C}=3$. (b) $\Gamma$ $=1.5, m_{C}=2$.

transmission. In the figures, the light source being on the same side as the camera, the front view shows a dark line, while the two side views show a bright line.

\section{Velocity measurements}

Velocity fields in a meridional plane $(x, z)$ were measured using a particle image velocimetry (PIV) system. For these measurements, the distance $H$ was set at $71 \mathrm{~mm}$, leading to an aspect ratio $\Gamma=H / R_{c}=2$. Small borosilicate particles, $11 \mu \mathrm{m}$ in diameter, are used as tracer, illuminated by a vertical laser sheet of thickness $b \simeq 0.5 \mathrm{~mm}$ produced by a double pulsed Nd:YAG laser (YAG-yttrium aluminum garnet). Images are acquired with a double-buffer high resolution camera $(1280 \times 1024$ pixels, 4096 gray levels $)$, synchronized with the laser at a rate of 1 frame pair per second.

The main difficulty for the PIV measurements in this geometry arises from the large azimuthal velocity component of the flow, which strongly constrains the time delay $\delta t$ between the two frames of a pair. The out-of-plane azimuthal velocity is of the order of $\Omega R$ and dominates the in-plane meridional components to be measured, which are of the order of $0.1 \Omega R$. In order to keep an acceptable number of common particles between the two frames of a pair, the time delay $\delta t$ has to be kept of the order of $b / \Omega R$. As a consequence, the particle displacement in the meridional plane is small of the order of $0.1 b \simeq 0.05 \mathrm{~mm} \simeq 1$ pixel. With azimuthal velocities of the order of $\Omega R \simeq 0.5-2 \mathrm{~m} \mathrm{~s}^{-1}$, the time delay $\delta t$ typically ranges from 0.5 to $2 \mathrm{~ms}$. Window sizes of (a)
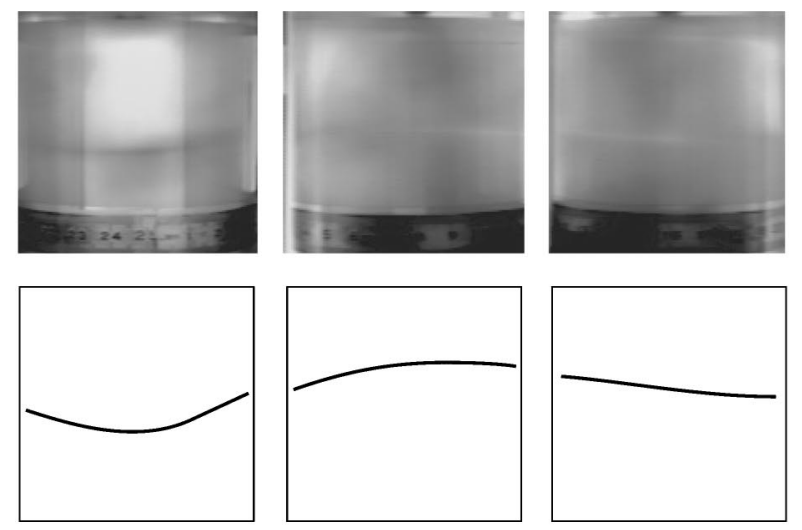

(b)
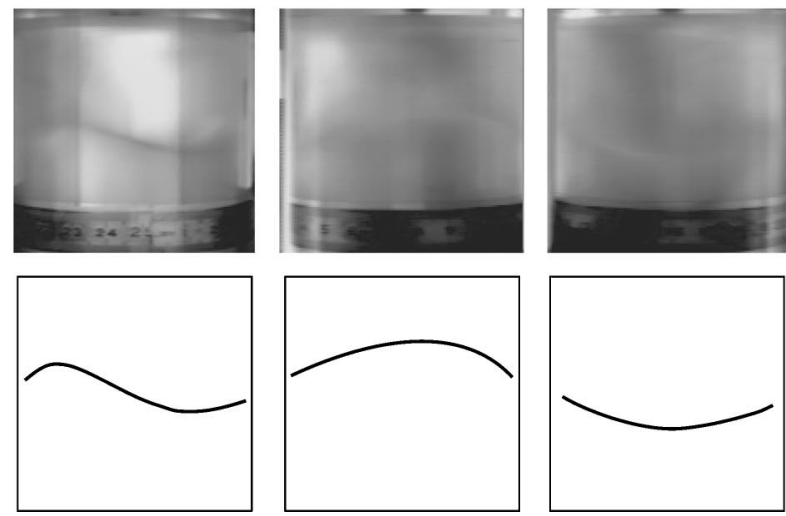

FIG. 3. Same as Fig. 2 with (a) $\Gamma=2, m_{C}=1$. (b) $\Gamma=2, m=2$.

$32 \times 32$ pixels, with an overlap of 16 pixels, are used for the PIV computations. The final velocity fields are defined on a $64 \times 64$ grid, with a resulting spatial resolution of $1 \mathrm{~mm}$. In the following figures, only one out of four velocity vectors is shown for clarity.

\section{REGIME DIAGRAM AND FLOW STATES}

Systematic measurements of the onset Reynolds numbers have been performed from visualization of the Iriodin flakes, for aspect ratios $\Gamma$ ranging between 0.5 and 2.5 . The regime diagram in Fig. 4 summarizes our observations in the plane of parameters $(\Gamma, \mathrm{Re})$. These onset Reynolds numbers have been obtained by slowly increasing the angular velocity and visually inspecting the light reflected and transmitted by the flakes.

The thresholds are found in excellent agreement with the numerical results of Nore et al., ${ }^{25}$ shown as the lower solid line, except for large $\Gamma$ where the experimental thresholds are slightly lower than the numerical ones, probably due to experimental noise which may slightly anticipate the bifurcation of the basic flow.

Examples of flow visualizations are given in Figs. 2 and 3. For low Reynolds numbers, the stable equatorial shear layer shows an exactly horizontal line at midheight, which becomes modulated as the Reynolds number is increased. The shear layer is sketched below the pictures to guide the eye. The corresponding mode can be easily determined as the number of extrema along the unfolded perimeter. The first three figures, Figs. 2(a), 2(b), and 3(a), obtained slightly 


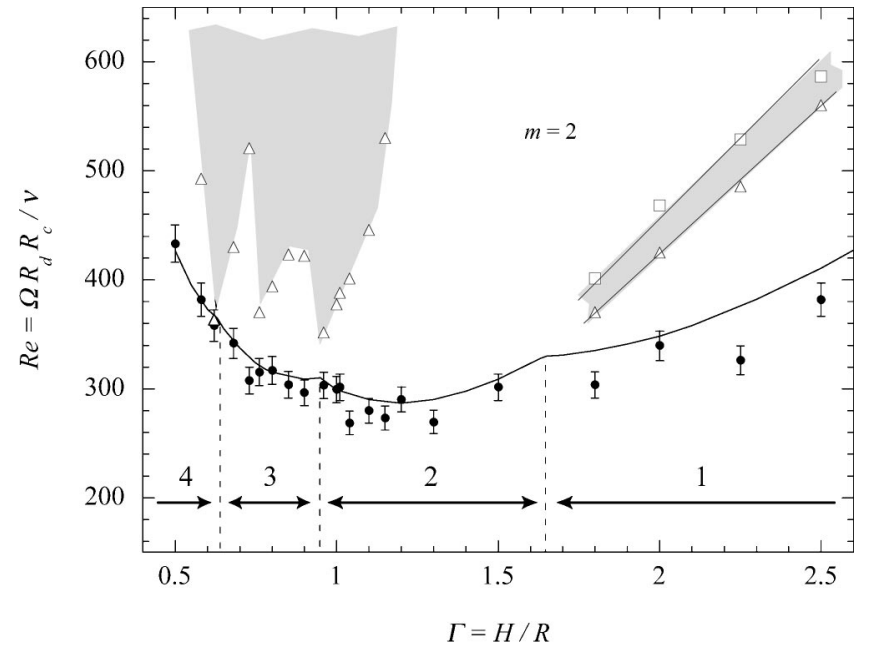

FIG. 4. Experimental regime diagram, obtained from visualization. steady nonaxisymmetric state with critical mode $m_{C}, \triangle$, time-dependent state, $\square$, steady nonaxisymmetric state with mode $m \neq m_{C}$. Error bars for $\triangle$ and $\square$ are the same as for $\bullet$. The gray region represents various unsteady states. - , numerical threshold, - - - lines indicating the codimension-two points, $\Gamma(3: 4)=0.63, \Gamma(2: 3)=0.95$, and $\Gamma(1: 2)=1.64$. The numbers above the horizontal arrows indicate the onset mode $m_{C}$.

above the onset, show the critical modes $m_{C}=3,2$, and 1 that appear for the aspect ratios $0.75,1.5$, and 2 , respectively. In the case $\Gamma=2$, another stationary mode $m=2$ is also obtained for higher Reynolds number, as shown in Fig. 3(b).

The first flow state found above the onset is always stationary, with an azimuthal modulation $m_{C}$ which decreases from 4 to 1 as $\Gamma$, i.e., the height, is increased. The experimentally observed onset modes fall into the expected regions shown by the horizontal arrows, delimited by the numerically determined codimension-two points sketched as vertical dashed lines in Fig. 4.

The subsequent states for larger Reynolds number depend strongly on the aspect ratio. For $\Gamma>\Gamma(1: 2)=1.64$, in addition to the critical mode $m_{C}=1$, a higher order stationary mode $m=2$ is also found when the Reynolds number is increased. These two modes, shown in Figs. 3(a) and 3(b) for $\Gamma=2$, correspond to what are called the mixed mode $M$ and the pure mode $P$, respectively, and will be described in detail in Sec. V. A narrow band of time-dependent states is experimentally observed between these two steady states. The upper and lower limits of this unsteady band are roughly straight lines that converge towards the codimension-two point at $\Gamma(1: 2)$, as predicted theoretically ${ }^{1,2,10}$ and confirmed numerically. ${ }^{25}$ In this band, two time-dependent states, traveling waves (TW), and near-heteroclinic cycles (Het) are experimentally observed, but they are not easy to distinguish from the visualizations. These two states will be described in detail in Sec. V for $\Gamma=2$.

For the range of aspect ratios $1.2<\Gamma<1.5$, in the $m_{C}$ $=2$ region, the flow remains stationary up to the highest Reynolds number investigated, $\operatorname{Re} \simeq 600$. Lower aspect ratios, $\Gamma \leqslant 1.2$, for which $m_{C} \geqslant 2$, show complex unsteady states. Traveling waves with strong nonlinear interactions, sometimes leading to intermittent vortex pairing, may be observed. The lower limit of this unsteady region is somewhat

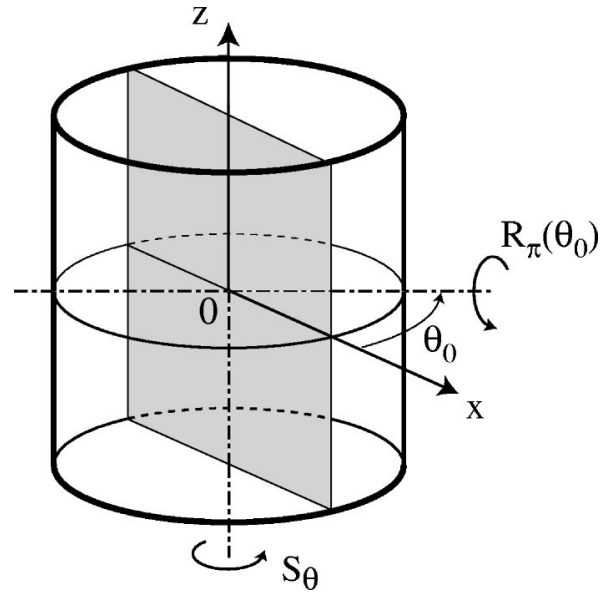

FIG. 5. Sketch of the two symmetries, $S_{\theta}$ and $R_{\pi}\left(\theta_{0}\right)$, of the flow between exactly counterrotating disks. The meridional plane where the PIV measurements are performed defines the $(x, z)$ plane, in gray.

scattered, sometimes closely approaching the critical curve, in particular, near the codimension-two points at $\Gamma(3: 4)$ $=0.63$ and $\Gamma(2: 3)=0.95$. No upper limit for this region is observed, at least for $\operatorname{Re}<600$. The complex flow states in this region could result from resonance between modes $m$ $\geqslant 2$, and have not yet been explored.

\section{SYMMETRIES AND FLOW STATES FOR $\Gamma=2$}

In order to obtain more insight into the sequence of bifurcations and the mode competition that takes place as the Reynolds number is increased, a careful analysis of the symmetries of the flow is essential. A complete description of these symmetries can be found in Nore et al., ${ }^{19}$ and they are only briefly presented here.

\section{A. Symmetries of the exactly counterrotating flow}

The base flow produced in a cylinder driven by the rotation of the top and bottom disks is axisymmetric, i.e., invariant under rotation by any angle $\theta$ about the cylinder axis, which we denote by $S_{\theta}$ (see Fig. 5). The present case of exactly counterrotating disks is unique in possessing an additional symmetry of rotation of $\pi$ about any horizontal axis in the equatorial plane. We denote this rotation by $R_{\pi}\left(\theta_{0}\right)$, where $\theta_{0} \in[0, \pi)$ is the direction of the horizontal axis. This additional symmetry is equivalent to combined reflections in $\theta=\theta_{0}$ and in $z=0$. The main point is that rotation about the $z$ axis and about a horizontal axis do not commute, and hence, the group generated by the operators $S_{\theta}$ and $R_{\pi}\left(\theta_{0}\right)$ is isomorphic to $O(2)$. This symmetry group $O(2)$ is especially important for the sequence of bifurcations present in this system, and is, in particular, the key ingredient for the heteroclinic cycles that take place between two rotated modes $m=2$ at $\Gamma=2$.

In the present experiment, only the velocity field in a $(x, z)$ meridional plane, which defines by convention the origin $\theta=0$, is measurable by PIV (see Fig. 5). It is therefore of interest to consider the restriction of the above threedimensional symmetries to this meridional plane. Note that, 
TABLE I. Symmetries of the various flow states and corresponding numerical and experimental critical Reynolds numbers for $\Gamma=2$. ( $\left.{ }^{*}\right)$ These modes may also be invariant under $s_{x}$ and $s_{0}$ for special values of $\theta_{0}$ (see text).

\begin{tabular}{|c|c|c|c|c|}
\hline State & Symmetry & $\begin{array}{c}\text { Symmetry } \\
\text { in }(x, z) \\
\text { plane }\end{array}$ & $\begin{array}{l}\text { Numerical } \\
\text { threshold }\end{array}$ & $\begin{array}{c}\text { Experimental } \\
\text { threshold }\end{array}$ \\
\hline Basic state & $S_{\theta}, R_{\pi}\left(\theta_{0}\right)$ & $s_{z}, s_{x}$ & $\ldots$ & $\ldots$ \\
\hline Mixed mode $(M)$ & $R_{\pi}\left(\theta_{0}\right)$ & $\left(^{*}\right)$ & 349.0 & $350 \pm 4$ \\
\hline Traveling waves (TW) & $\ldots$ & $\ldots$ & 411.6 & $420 \pm 4$ \\
\hline Modulated waves (MW) & $\ldots$ & $\ldots$ & 427.3 & Not observed \\
\hline Heteroclinic cycles (Het) & $\ldots$ & $\ldots$ & 427.4 & $450 \pm 8$ \\
\hline Pure mode $(P)$ & $S_{\pi}, R_{\pi}\left(\theta_{0}\right)$ & $s_{z},\left(^{*}\right)$ & 452 & $480 \pm 4$ \\
\hline
\end{tabular}

in this restriction, we consider only the velocity components within the meridional plane, and not the component perpendicular to it.

Consider first states which are axisymmetric. In the meridional plane, these states are invariant under reflection in the $z$ axis, acting on coordinates as $(x, z) \rightarrow(-x, z)$, which we denote by $s_{z}$. This is also the case for even wavenumber states, those which are invariant under discrete rotations $S_{\theta}$ with $\theta=2 \pi / m$ for even $m$. Consider now states which are invariant under some reflection $R_{\pi}\left(\theta_{0}\right)$, where $\theta_{0}$ is an arbitrary angle chosen by the system. These states are not necessarily symmetric in the $(x, z)$ meridional plane where the PIV measurements are performed, unless $\theta_{0}$ takes on one of two special values. If $\theta_{0}$ is along the $x$ axis $\left(\theta_{0}=0\right)$, then invariance under $R_{\pi}\left(\theta_{0}\right)$ reduces to reflection in the $x$ axis, denoted by $s_{x}$ and acting as $(x, z) \rightarrow(x,-z)$. If $\theta_{0}$ is normal to the plane $\left(\theta_{0}=\pi / 2\right)$, then invariance under $R_{\pi}\left(\theta_{0}\right)$ is the central symmetry relative to the origin, denoted by $s_{0}$, which is equivalent to combined reflection in $x$ and $z$, i.e., $s_{0}=s_{x} s_{z}$. These symmetries in the meridional plane, $s_{z}, s_{x}$, and $s_{0}$, are especially useful as they provide a convenient way of identifying the flow patterns obtained by PIV in Sec. V.

\section{B. Numerical results for $\Gamma=2$}

The particular case of the aspect ratio $\Gamma=2$ shows the following sequence of bifurcations as the Reynolds number is increased: ${ }^{19}$ basic flow; steady state with $m=1$, called mixed mode $M$; traveling waves; modulated traveling waves; near-heteroclinic cycles; and steady states with $m=2$, called pure mode $P$. The symmetries and the critical Reynolds numbers of these states are described here, and are summarized in Table I.

For low Reynolds number, the basic state inherits the $O(2)$ symmetry from the geometric configuration. In the meridional plane, it is thus invariant under $s_{z}$ and $s_{x}$. For $\operatorname{Re}$ $>349.0$, this basic state loses stability to the $m=1$ mode, giving rise to a stationary state with one elliptic point in the equatorial plane and a diametrically opposite hyperbolic point. This state is called the mixed mode $M$, because the nonlinear interactions produce both even and odd harmonics. The other stationary bifurcated flow is obtained for $\mathrm{Re}$ $\geqslant 452$. It is a $m=2$ mode and consists of two radial corotating vortices in the equatorial plane, located on two diametrically opposite elliptic points $E$ and $E^{*}$, and separated by two
(P)
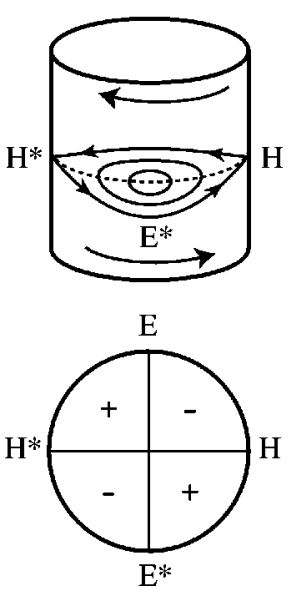

$\left(\mathrm{P}^{\prime}\right)$
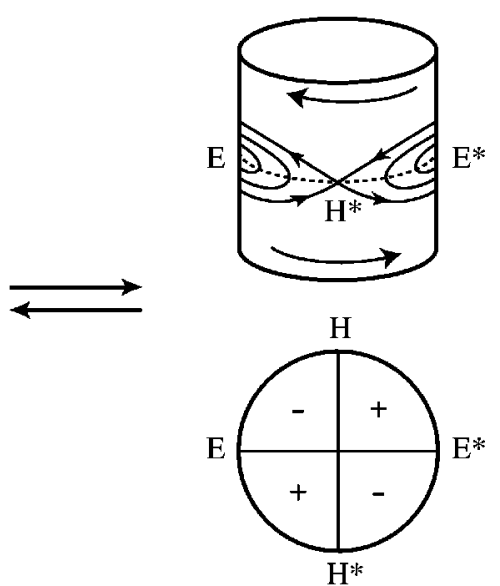

FIG. 6. (Bottom) Sketch of vertical velocity variations in the equatorial plane of the pure modes $P$ and $P^{\prime}$. The $+(-)$ sign designates upward (downward) vertical velocities. The heteroclinic cycle connects $P$ and $P^{\prime}$, related by rotation of $\pi / 2$. The radial vortices are centered on the diametrically opposite elliptic points $\left(E, E^{*}\right)$ and are located at right angle from the hyperbolic points $\left(H, H^{*}\right)$. (Top) Perspective representations of the two corotating vortices separated by the two hyperbolic points $\left(H, H^{*}\right)$.

hyperbolic points $H$ and $H^{*}$. Two orientations of this state are sketched in Fig. 6. This state is called the pure mode $P$ since only even harmonics are present. Both the mixed mode $M$ and the pure mode $P$ break the invariance under $S_{\theta}$, while preserving the invariance under $R_{\pi}\left(\theta_{0}\right)$. As seen in the preceding section, this invariance does not lead to any particular symmetry in the meridional plane where the PIV measurements are performed, except for special orientations of $\theta_{0}$ which lead to the horizontal reflection symmetry $s_{x}$ or the central symmetry $s_{0}$. In addition, since it involves only even Fourier components, the pure mode $P$ is also invariant under $S_{\theta}$ for $\theta=\pi$, and is consequently invariant under $s_{z}$ in the meridional plane.

For intermediate Reynolds numbers between these two steady states, $411.6 \leqslant \operatorname{Re} \leqslant 452$, three types of timedependent states have been numerically reported. For 411.6 $\leqslant \operatorname{Re} \leqslant 427.3$, right or left TW are observed, which break both the $S_{\theta}$ and the $R_{\pi}\left(\theta_{0}\right)$ symmetries. These waves become modulated in a very narrow range of Reynolds numbers, $427.3 \leqslant \operatorname{Re} \leqslant 427.4$. For $\operatorname{Re}>427.4$, the solution follows an attracting near-heteroclinic cycle which links two unstable pure states $P$ and $P^{\prime}$, obtained by rotation of $\pi / 2$ about the cylinder axis (see Fig. 6). These cycles are stable up to $\operatorname{Re}$ $=452$, where the steady pure mode $P$ becomes stable. Although the flow during the near-heteroclinic cycle presents no exact spatial symmetry, during the quasistationary phases in the neighborhood of the states $P$ and $P^{\prime}$ it does inherit the symmetries of the pure mode state, i.e., invariance under $s_{z}$ (because of the even harmonic content) and possibly the invariances under $s_{x}$ and therefore also $s_{0}$ (for special orientations of $\theta_{0}$ ).

The numerically computed near-heteroclinic cycles found in Nore et al. ${ }^{19}$ are characterized by periodic oscillations between pure mode states $P$ and $P^{\prime}$ separated by rapid changes through odd-wavenumber-dominated states. The period is controlled by the equal time $\tau$ spent in the neighbor- 
hoods of $P$ and $P^{\prime}$. This duration $\tau$ increases from 550 up to 900 rotation periods when the Reynolds number Re increases from 428 to 435 . Two distinct heteroclinic type regimes were observed in the simulations: a two-plateau and a four-plateau oscillation. These two regimes originate from two possible paths connecting the two saddle points $P$ and $P^{\prime}$. Although no systematic conclusion could be reached in the simulations due to the prohibitive computation time, both the Reynolds number and the choice of the initial conditions seemed to play a role in favoring one of these two regimes.

\section{PIV ANALYSIS OF THE FLOW STATES FOR $\Gamma=2$}

Systematic measurements of the velocity fields in the meridional plane $(x, z)$ have been performed for $\Gamma=H / R_{c}$ $=2$, with the aim of further characterization of the nearheteroclinic cycles. In Table I, the experimentally observed flow states are summarized, and the corresponding critical Reynolds numbers are compared to the numerical ones.

\section{A. Steady states}

Figure 7 shows the meridional velocity field $\left(u_{x}, u_{z}\right)$ for the three different steady states observed at $\Gamma=2$ : (a) the basic state for $\operatorname{Re}<350 \pm 4$; (b) the mixed mode $M$ for $350 \pm 4<\operatorname{Re}<420 \pm 4$; (c) the pure mode $P$ for $\operatorname{Re}>480 \pm 4$. These velocity fields are time averaged over the whole run. The experimental thresholds, given in Table I, are in very good agreement with the numerical ones, with discrepancies that do not exceed $6 \%$.

Contour levels of the velocity $u_{x}$ are also shown, and are especially useful in depicting the symmetries of the various flow states. In the basic state, Fig. 7(a), the contour levels are even in $z$ and almost odd in $x$; the residual asymmetry in $x$ probably results from a slight misalignment of the laser sheet with the meridional plane, leading to a contamination from the large azimuthal component of the velocity. As shown in Sec. IV, this corresponds to invariance under the reflection symmetries $s_{x}$ and $s_{z}$, which traces back to the $O(2)$ symmetry of the basic state. The mixed mode $M$, Fig. 7(b), shows symmetry neither in $x$ nor in $z$, since the $R_{\pi}\left(\theta_{0}\right)$ symmetry for a mixed mode does not lead to any symmetry in an arbitrary meridional plane. The departure from reflection symmetry can be verified by carefully looking at the velocity vectors near the vertical axis of Fig. 7(b) which are not symmetric under $s_{0}$. In the pure mode $P$, Fig. 7(c), the vertical reflection symmetry $s_{z}$ of the contour levels is recovered, as the trace of the presence of only even harmonics.

It must be noted that the experimentally observed mixed and pure modes always show similar velocity fields in the meridional PIV plane, even for different initial conditions. In particular, the $s_{0}$ or $s_{x}$ symmetries are never observed for these flow patterns, as would be the case for a flow state invariant under the $R_{\pi}\left(\theta_{0}\right)$ symmetry with $\theta_{0}=0$ or $\pi / 2$. A geometric defect in the setup, probably the residual ellipticity of the cylindrical container (of about $0.7 \%$ ), is thought to be responsible for this systematic bias of the azimuthal symmetry breaking. This imperfection probably has no influence on the transition from the basic to the mixed state. However, by forcing the flow in a preferred direction, it may delay the (a) Basic

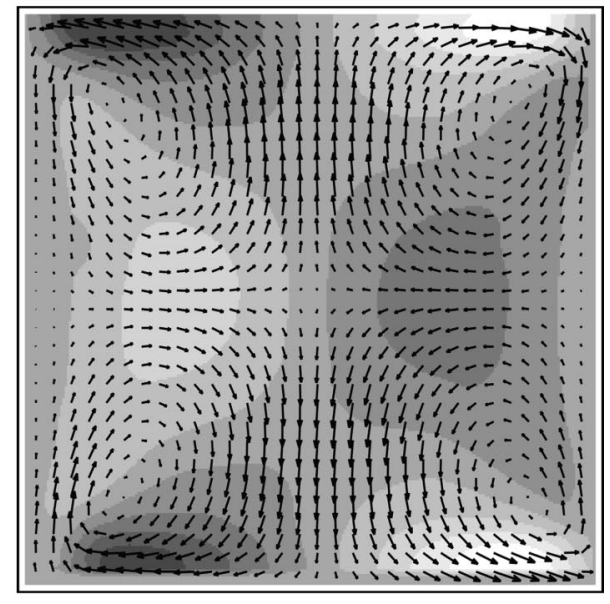

(b) Mixed M

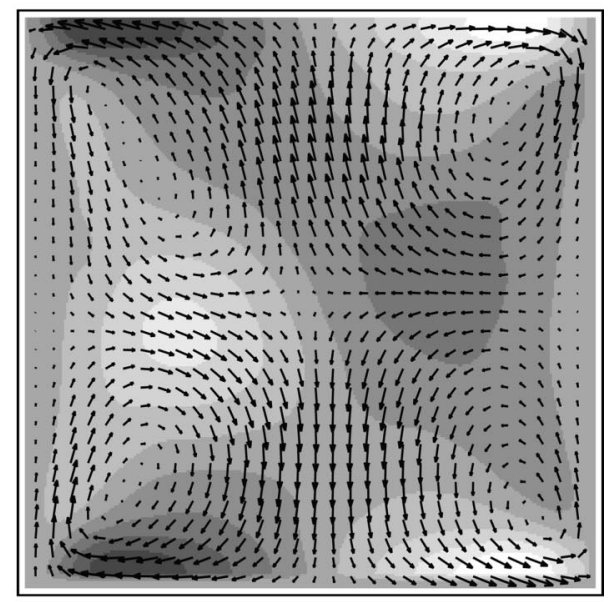

(c) Pure P

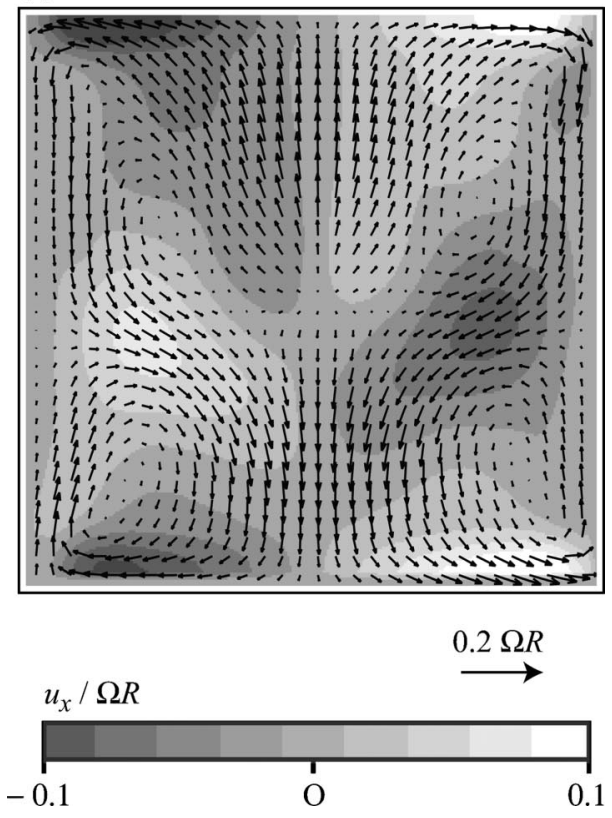

FIG. 7. Meridional velocity field $\left(u_{x}, u_{z}\right)$ and contour levels of $u_{x}$ of the three steady states. (a) Basic state at $\operatorname{Re} \simeq 280$, invariant under the horizontal and vertical reflection symmetries $s_{x}$ and $s_{z}$. (b) Mixed mode $M$ at $\operatorname{Re}$ $\simeq 415$, which does not show any invariance as can be verified on the arrows near the vertical axis. (c) Pure mode $P$ at $\operatorname{Re} \simeq 550$, invariant under the vertical reflection symmetry $s_{z}$. 


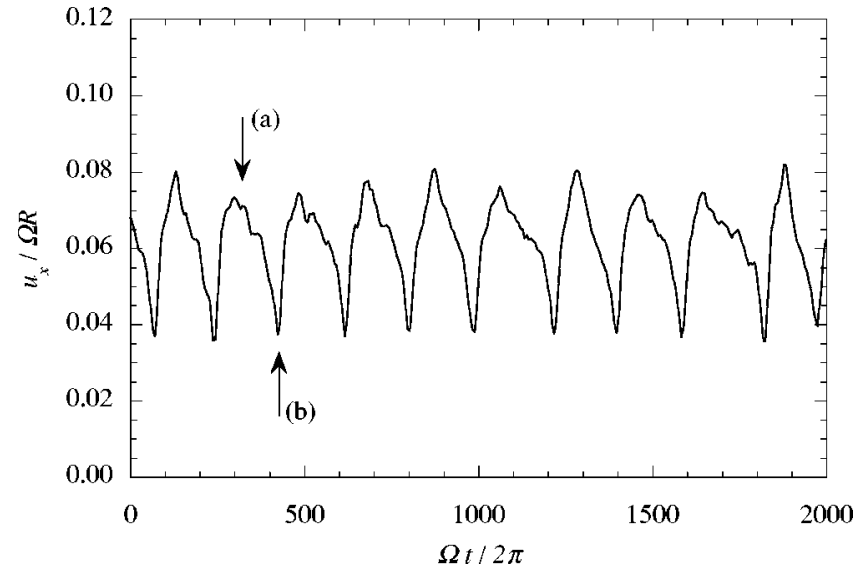

FIG. 8. Time series of $u_{x}(x=-R / 2, z=0)$ corresponding to the traveling waves $T W$ for $R e \simeq 440$. The labels (a) and (b) refer to the velocity fields in Fig. 9.

transitions toward the subsequent states. This may explain the above mentioned shifts, of the order of $6 \%$, between the numerical and the experimental thresholds (see Table I).

\section{B. Traveling waves}

The mixed mode state loses stability at $\operatorname{Re} \simeq 420 \pm 4$, giving rise to a first time-dependent state, as illustrated by the time series in Fig. 8 for $R e \simeq 440$. This figure shows nearly periodic oscillations, with a period of $190 \pm 15$ rotations, of the horizontal velocity $u_{x}$, measured at the location $(x, z)$ $=(-R / 2,0)$.

This first time-dependent state can be identified as the TW state, by inspecting the velocity fields in Fig. 9. These fields correspond to the two characteristic phases that are labeled (a) and (b) in the time series. They are more noisy than those for the steady states, as they have been averaged over only a few instantaneous velocity fields. As expected, these fields do not present any particular symmetry, since the traveling waves break both the $S_{\theta}$ and the $R_{\pi}\left(\theta_{0}\right)$ symmetries. It must be noted, however, that Fig. 9(b) appears to be similar to the steady mixed mode shown in Fig. 7(b), which is invariant under $R_{\pi}\left(\theta_{0}\right)$. This resemblance indicates that the asymmetric (under $R_{\pi}$ ) component of the traveling wave state is indeed weak.

The theoretical analysis shows that these traveling waves originate from a drift pitchfork bifurcation, ${ }^{26}$ and thus have infinite period at onset. In the range of Reynolds numbers
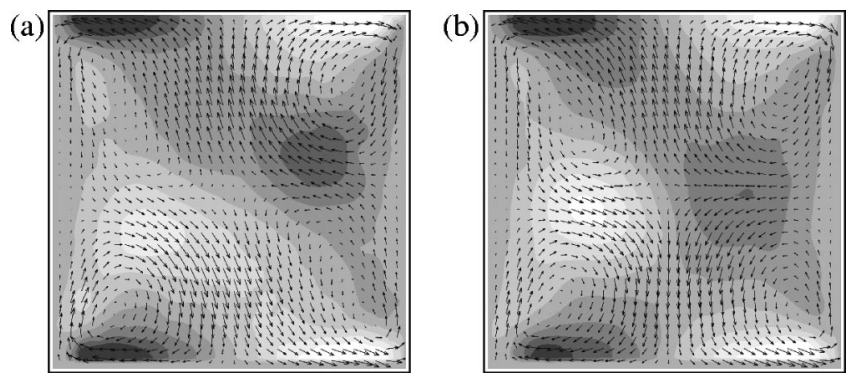

FIG. 9. Velocity fields of the traveling waves TW for $\mathrm{Re} \simeq 440$ (see Fig. 8). The velocity scale and contour levels gray scale are as that in Fig. 7.
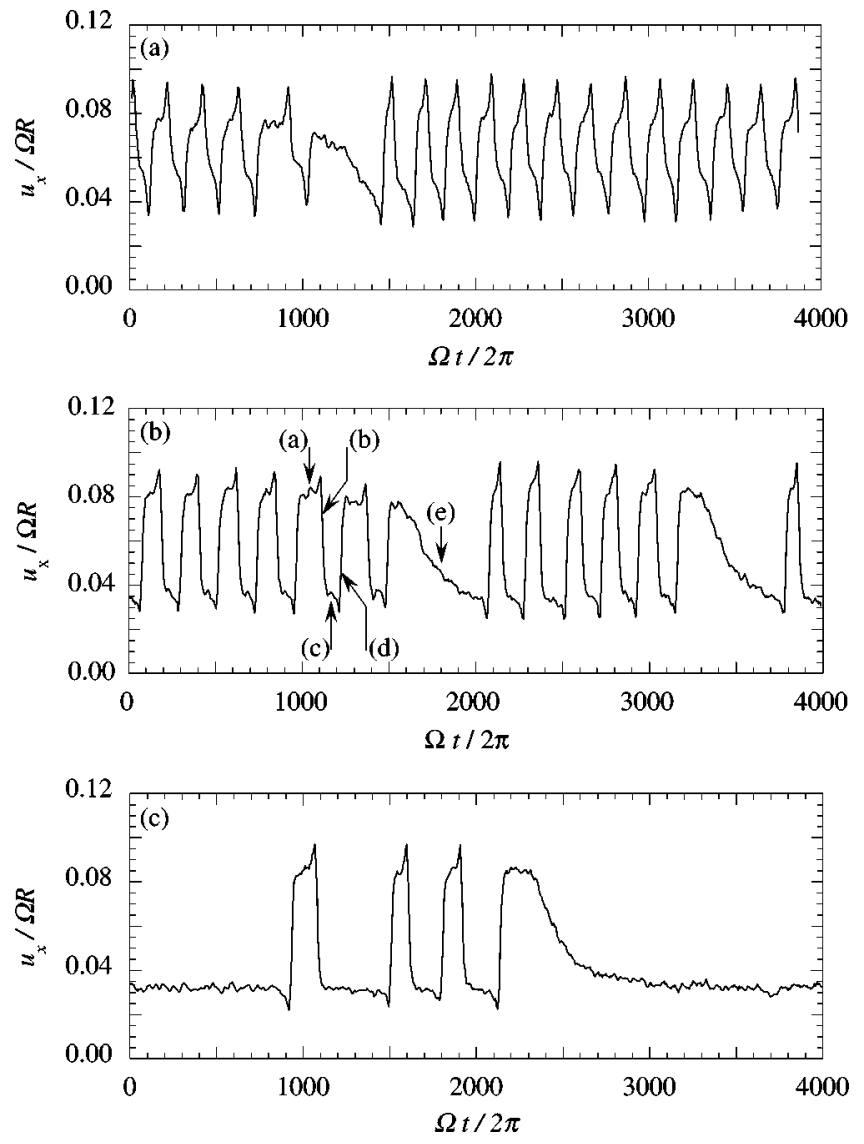

FIG. 10. Time series of $u_{x}(x=-R / 2, z=0)$, corresponding to the nearheteroclinic cycle state (Het), for $\mathrm{Re} \simeq 452,469$, and 478 . The labels (a)-(e) in the second time series refer to the velocity fields in Fig. 11.

where the traveling waves are observed, the measured period remains of the order of $190 \pm 15$ rotations. Numerically, the period was found to decrease as the Reynolds number is increased as predicted by theory. However, because of the small range of Reynolds number where this pattern can be observed, no measurable change in the period has been detected experimentally. Note also that the modulated waves (MW), which were observed only in a very narrow range of Reynolds numbers in the simulations, $427.3 \leqslant \operatorname{Re} \leqslant 427.4$ (see Table I), have not been observed in the experiments. The very fine range of Reynolds number where they should be observed is well beyond the experimental uncertainty. Moreover, the geometrical differences between the experimental and the numerical configurations, in particular, the cylinderto-disk radius ratio $R_{c} / R_{d}$ of 1.03 instead of 1 , cannot allow us to expect a perfect agreement for the observed flow states and their critical Reynolds numbers.

\section{Near-heteroclinic cycles}

When the Reynolds number is increased above 450, the time series cease to show a strictly periodic behavior. Instead, irregular oscillations between two states, punctuated by slow relaxation phases toward a third state, are observed, as shown by the time series in Fig. 10 obtained for increasing Reynolds number, $\operatorname{Re} \simeq 452,469$, and 478. As the Reynolds 
(a)

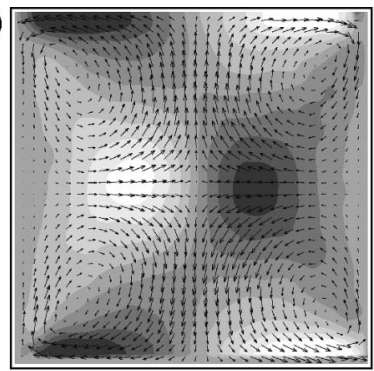

(c)

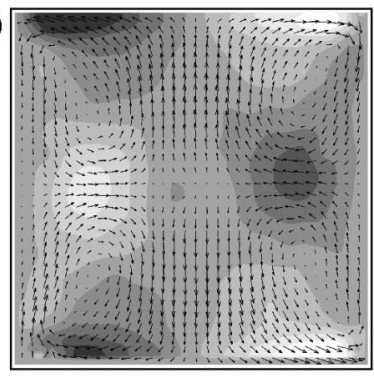

(b)

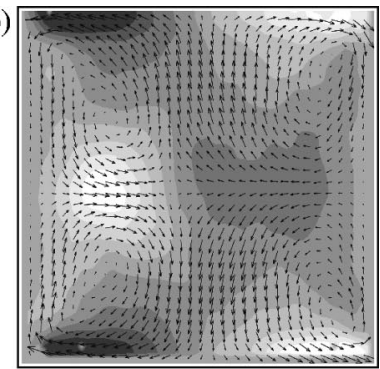

(d)

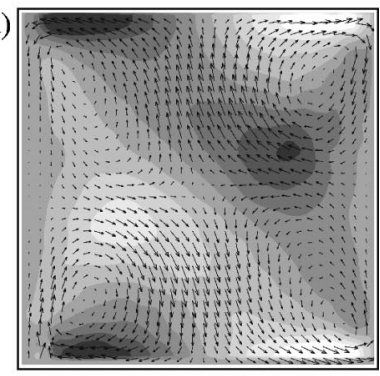

(e)

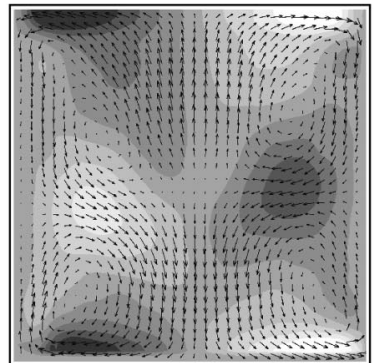

FIG. 11. Velocity fields of the near-heteroclinic cycle state (Het) for Re $\simeq 469$. The fields (a) and (c), approximately invariant under the vertical reflection symmetry $s_{z}$, correspond to the pure states $P$ and $P^{\prime}$ (plateaux of approximately constant $u_{x}$ in Fig. 10). The fields (b) and (d) are instantaneous velocity fields corresponding to the spikes in Fig. 10 that connect the plateaux (a) and (c). The field (e) in the long relaxation phase is the pure mode favored by an experimental defect resembling the pure mode in Fig. $7(\mathrm{c})$.

number approaches the upper bound, $\mathrm{Re} \simeq 480$, the relaxation phases are found to be longer and more frequent.

The velocity fields corresponding to the different phases of the second time series, labeled (a)-(e), are shown in Fig. 11. The fields (a) and (c) correspond, respectively, to the upper and the lower plateaux of the oscillations, the rapid changes between them are denoted by (b) and (d), and the last field (e) corresponds to the slow relaxation phase.

Visual inspection of the symmetries of the fields (a)-(d) allows us to identify this second time-dependent state as the Het state connecting the two unstable pure modes $P($ a) and $P^{\prime}(\mathrm{c})$. The two velocity fields (a) and (c) are indeed approximately invariant under the vertical reflection symmetry $s_{z}$, as expected for pure modes $m=2$ with only even harmonics. This property distinguishes without ambiguity the heteroclinic cycle state from the traveling wave state (see Fig. 9), which is not invariant under $s_{z}$. In addition, the two fields (a) and (c) appear to be also approximately invariant under $s_{x}$ (and hence under $s_{0}=s_{x} s_{z}$ ), so that the vertical velocity component is almost zero on the equatorial line $z=0$. The comparison with the sketches of the equatorial plane in Fig. 12, where the sign of $u_{z}$ is shown, indicates that the PIV plane
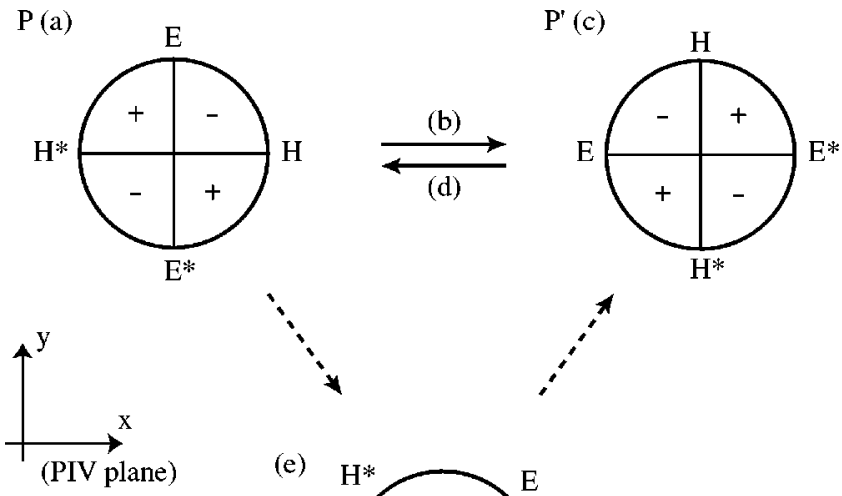

(e)

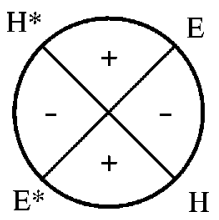

FIG. 12. Sketch of the experimental near-heteroclinic cycle connecting the two pure modes $P\left(\right.$ a) and $P^{\prime}(\mathrm{c})$ [double arrow (b) and (d)], punctuated by excursions toward the favored pure mode (e) oriented at $45^{\circ}$ from the diameters $E E^{*}$ and $H H^{*}$ (dashed arrows). The labels (a)-(e) refer to those of Figs. 10 and 11. The PIV plane is aligned with the $x$ axis.

where the measurements are performed appears to be approximately aligned either with the two hyperbolic points $H$ and $H^{*}$ [Fig. 12(a)], or with the two elliptic points $E$ and $E^{*}$ [Fig. 12(c)]. This confirms that the two unstable states $P$ and $P^{\prime}$ of the cycle are related by a $\pi / 2$ rotation. ${ }^{27}$ The fields corresponding to the rapid changes between $P$ and $P^{\prime}$ shown in Figs. 11(b) and 11(d) are, as expected, states of mixed (even and odd) harmonic content that connect the two pure modes. The field (b) is approximately invariant under the $s_{x}$ symmetry, while the field (d) is invariant under the $s_{0}$ symmetry. The characteristic time of these transient phases is of about $20 \pm 5$ rotations, which is of the order of the time scale of the advection by the meridional components of the velocity.

Finally, the last field in Fig. 11(e), which corresponds to the slow relaxation phases that appear intermittently during the cycles (a)-(d), makes the above description in terms of a heteroclinic cycle more complex. This additional state, which is invariant under $s_{z}$ but not under $s_{x}$, resembles the steady pure mode observed at larger Reynolds numbers [see Fig. 7(c)], with two large counterrotating vortices in the upper cylinder half and two smaller ones in the lower half. The negative values of $u_{z}$ along the equatorial line $z=0$ indicate that the PIV plane now falls in between the two diameters $E E^{*}$ and $H H^{*}$ [see Fig. 12(e)], i.e., at $\approx 45^{\circ}$ from the pure states $P$ and $P^{\prime}$ of the cycle. As noted in Sec. V A, this particular orientation of the steady pure mode seems to be favored by a slight asymmetry in the experimental setup. This suggests that the imperfection of the experiment, although weak, has a deep influence on the observed nearheteroclinic cycles, and would be, in particular, responsible for the relaxation phases that intermittently interrupt the oscillations between $P$ and $P^{\prime}$ (see the dashed arrows in Fig. 12). 


\section{Plateau durations of the near-heteroclinic cycles}

For Reynolds numbers close to the lower bound where the near-heteroclinic cycle state is found, $\operatorname{Re} \simeq 450$, apart from the slow relaxation phases toward the favored pure mode (e), the plateau duration $\tau$ is approximately constant of the order of 100 rotations. These plateau durations become uneven as the Reynolds number is increased, with a mean duration $\langle\tau\rangle$ that gradually increases [200 rotations for Re $\simeq 469$ in Fig. 10(b)]. In addition, the relaxation phases (e) are longer and more frequent, and are preceded by a gradual increase of the plateau durations, as clearly seen in Fig. 10(b). This scenario of a gradual increase of the plateau durations interrupted by an excursion toward the favored pure mode (e) and back to the plateau oscillations (a)-(d) is observed in most of the time series.

The broad distribution of plateau durations at fixed Reynolds number is a genuine property of the approach toward a heteroclinic cycle in the presence of noise. ${ }^{28}$ The plateau duration is indeed controlled by the time spent by the system in the neighborhoods of the two unstable states $P$ and $P^{\prime}$. These two states being saddle points, there is no intrinsic time scale for this approach, which should tend to infinity in the absence of noise. ${ }^{5}$ When a certain amount of noise $\epsilon$ $\ll 1$ is present, the system approaches one of the saddle points along its attracting direction as $\exp \left(-\lambda_{s} t\right)$ down to a distance of the order of $O(\epsilon)$. Meanwhile, it moves away along the repulsing direction as $\exp \left(\lambda_{u} t\right)$, until it reaches a distance $\sim O(1)$ at which it escapes from the saddle point. Here $\lambda_{s}$ and $\lambda_{u}$ are the absolute values of the leading eigenvalues in the stable and unstable directions, respectively. As a consequence, the mean plateau duration is given by ${ }^{28}$

$$
\langle\tau\rangle \simeq \frac{1}{\lambda_{u}}|\ln \epsilon| .
$$

The distribution of $\tau$ cannot be deduced from this crude description and depends on the statistical properties of the noise. The observed increase of the mean plateau duration $\langle\tau\rangle$ as the Reynolds number is increased presumably does not originate from the noise of the system, which should not evolve significantly over this small range of Reynolds numbers $\operatorname{Re} \in[450,480]$, but is more likely due to a Re dependence of the unstable eigenvalue $\lambda_{u}$. This eigenvalue is indeed expected to vanish at $\operatorname{Re} \simeq 480$, when the heteroclinic cycle branch disappears and the pure mode branch becomes stable, which should lead to an infinite mean plateau duration.

Note that an ideal heteroclinic cycle without any noise nor geometrical defect should lead to infinitely increasing plateau durations. ${ }^{9,10}$ This could explain the gradual increase of the plateau durations before the relaxation phases (e) observed in most time series. In contrast, the simulations of Nore et al. ${ }^{19}$ only showed strictly periodic cycles with a fixed finite period. In the numerics, various sources of noise, such as round-off errors and temporal discretization, by systematically bringing the system to the same distance from the saddle points, may have a saturating effect on the period of the cycles. In our experiment, both the noise and the geometrical bias seem to play an important role in the observed near-heteroclinic cycles. First, the presence of noise may prevent the system from staying an infinite time close to one of the unstable states. Second, the geometrical defect seems to enable the system to explore another path toward the favored pure mode state. However, a systematic characterization of the duration statistics is well beyond the available data, and would require much longer acquisition times in a perfectly stable system.

\section{CONCLUSION AND DISCUSSION}

We have experimentally investigated the dynamics of the exactly counterrotating von Kármán swirling flow at low Reynolds numbers and for different height-to-radius aspect ratios $\Gamma \in[0.5,2.5]$. Visualizations based on Iriodin flakes have demonstrated that the first instability gives rise to stationary vortex patterns, the number of vortices increasing as $\Gamma$ decreases. The experimental thresholds are in good agreement with the numerical findings. ${ }^{25}$ A survey of the subsequent flow states that take place for higher Reynolds numbers has been performed. In the particular case of $\Gamma=2$, all flow states observed in the numerical simulations have been recovered except for a single state, the modulated traveling waves, which occur numerically in a very narrow range of Reynolds numbers.

For the first time, experimental evidence is given for the existence of robust heteroclinic cycles in this flow, as predicted by the numerical simulations of Nore et al. ${ }^{19}$ for $\Gamma$ $=2$. This regime originates from a resonance between two stationary states, $m=1$ and $m=2$, in the presence of the $O(2)$ symmetry. It is worth pointing out that the existence of steady bifurcated states and heteroclinic cycles depends strongly on the exact counterrotation of the top and bottom disks. For a rotation ratio different from -1 , the $O(2)$ symmetry would have been broken, and the system would have only kept the axisymmetry $S O(2)$. Therefore, the patterns would have generically rotated and the heteroclinic cycles would have been destroyed by this symmetry breaking, see, e.g., Porter and Knobloch. ${ }^{29}$ The experimental setup used in the present study, where a single dc motor drives the two disks, constrains the rotation ratio to be exactly -1 , thus allowing us to experimentally observe the steady bifurcated states and the heteroclinic cycles.

Perhaps the most interesting observation concerning the near-heteroclinic cycles is the broad distribution of the time spent in the vicinity of each unstable pure mode state and the excursions toward another pure mode favored by a geometrical imperfection. While for low Reynolds numbers approximately periodic oscillations are found, uneven periods are encountered as the Reynolds number is increased, showing increasing plateau durations followed by long relaxation phases toward the favored pure mode state. These oscillations differ from the strictly periodic near-heteroclinic cycles reported in the numerical simulations of Nore et al. ${ }^{19}$ In our experimental realization of the von Kármán flow, both noise and geometrical defects, although weak, play a role in the observed irregular oscillations, and only further investigation can show which of the two is of primary importance. 


\section{ACKNOWLEDGMENTS}

This work was benefited from fruitful discussions with Yves Couder, Olivier Le Maître, Marc Rabaud, and Laurette Tuckerman and from very interesting suggestions from the referees.

${ }^{1}$ J. Guckenheimer and P. Holmes, "Structurally stable heteroclinic cycles," Math. Proc. Cambridge Philos. Soc. 103, 189 (1988).

${ }^{2}$ M. Golubitsky, I. Stewart, and D. Schaeffer, Singularities and Groups in Bifurcation Theory, Applied Mathematics Sciences Vol. 69 (Springer, New York, 1988).

${ }^{3}$ M. Krupa and I. Melbourne, "Asymptotic stability of heteroclinic cycles in systems with symmetry," Ergod. Theory Dyn. Syst. 15, 121 (1995).

${ }^{4}$ M. Krupa, "Robust heteroclinic cycles," J. Nonlinear Sci. 7, 129 (1997).

${ }^{5}$ F. H. Busse and K. E. Heikes, "Convection in a rotating layer: A simple case of turbulence," Science 208, 173 (1980).

${ }^{6}$ G. Küppers and D. Lortz, "Transition from laminar convection to thermal turbulence in a rotating fluid layer," J. Fluid Mech. 35, 609 (1969).

${ }^{7}$ R. M. May and W. J. Leonard, "Nonlinear aspects of competition between three species," SIAM J. Appl. Math. 29, 243 (1975).

${ }^{8}$ C. A. Jones and M. R. E. Proctor, "Strong spatial resonance and travelling waves in Bénard convection," Phys. Lett. A 121, 224 (1987).

${ }^{9}$ M. R. E. Proctor and C. A. Jones, "The interaction of two spatially resonant patterns in thermal convection. Part 1. Exact 1:2 resonance," J. Fluid Mech. 188, 301 (1988).

${ }^{10}$ D. Armbruster, J. Guckenheimer, and P. Holmes, "Heteroclinic cycles and modulated traveling waves in systems with $\mathrm{O}(2)$ symmetry," Physica D 29, 257 (1988).

${ }^{11}$ S. Cox, "Mode interactions in Rayleigh-Bénard convection," Physica D 95, 50 (1996).

${ }^{12}$ I. Mercader, J. Prat, and E. Knobloch, "Robust heteroclinic cycles in twodimensional Rayleigh-Bénard convection without Boussinesq symmetry," Int. J. Bifurcation Chaos Appl. Sci. Eng. 12, 2501 (2002).

${ }^{13}$ N. Aubry, P. Holmes, J. L. Lumley, and E. Stone, "The dynamics of coherent structures in the wall region of a turbulent boundary layer," J. Fluid Mech. 92, 115 (1988).

${ }^{14}$ P. Holmes, J. L. Lumley, and G. Berkooz, Turbulence, Coherent Structures, Dynamical Systems and Symmetry (Cambridge University Press, Cambridge, 1996)

${ }^{15}$ I. G. Kevrekidis, B. Nicolaenko, and J. C. Scovel, "Back in the saddle again: A computer assisted study of the Kuramoto-Sivashinsky equation," SIAM J. Appl. Math. 50, 760 (1990).
${ }^{16}$ Y. Hu, W. Pesch, G. Ahlers, and R. E. Ecke, "Convection under rotation for Prandtl numbers near 1: Küppers-Lortz instability," Phys. Rev. E 58, 5821 (1998).

${ }^{17}$ E. Stone, M. Gorman, M. El Hamdi, and K. A. Robbins, "Identification of intermittent ordered patterns as heteroclinic connections," Phys. Rev. Lett. 76, 2061 (1996).

${ }^{18}$ A. Palacios, G. H. Gunaratne, and M. Gorman, "Cellular pattern formation in circular domains," Chaos 7, 463 (1997).

${ }^{19}$ C. Nore, L. S. Tuckerman, O. Daube, and S. Xin, "The 1:2 mode interaction in exactly counter-rotating von Kármán swirling flow," J. Fluid Mech. 477, 51 (2003).

${ }^{20}$ F. Marques, J. M. Lopez, and J. Shen, "Mode interactions in an enclosed swirling flow: A double Hopf bifurcation between azimuthal wavenumbers 0 and 2," J. Fluid Mech. 455, 263 (2002).

${ }^{21}$ J. M. Lopez, J. E. Hart, F. Marques, S. Kittelman, and J. Shen, "Instability and mode interactions in a differentially driven rotating cylinder," J. Fluid Mech. 462, 383 (2002).

${ }^{22}$ F. Marques, A. Yu. Gelfgat, and J. M. Lopez, "Tangent double Hopf bifurcation in a differentially rotating cylinder flow," Phys. Rev. E 68, 016310 (2003)

${ }^{23}$ F. Moisy, O. Doaré, T. Pasutto, O. Daube, and M. Rabaud, "Experimental and numerical study of the shear layer instability between two counterrotating disks," J. Fluid Mech. 507, 175 (2004).

${ }^{24}$ J. M. Lopez and F. Marques, "Mode competition between rotating waves in a swirling flow with reflection symmetry," J. Fluid Mech. 507, 265 (2004).

${ }^{25}$ C. Nore, M. Tartar, O. Daube, and L. S. Tuckerman, "Survey of instability thresholds of flow between exactly counter-rotating disks," J. Fluid Mech. 511, 45 (2004).

${ }^{26} \mathrm{P}$. Coullet and G. Iooss, "Instabilities of one-dimensional cellular patterns," Phys. Rev. Lett. 64, 866 (1990).

${ }^{27}$ Comparisons with the numerical computations [see Fig. 16(c) in the paper of Nore et al. (Ref. 19)] allow us to further identify the orientation of the observed states $P$ and $P^{\prime}$. The velocity field of the state $P$ [Fig. 11(a)] shows a unique hyperbolic point at the center $(x, z)=(0,0)$, indicating that the two peripherical hyperbolic points $H$ and $H^{*}$ are close to the PIV plane where the measurements are performed [see Fig. 12(a)]. On the other hand, two hyperbolic points can be seen near the cylinder axis for the state $P^{\prime}$ [Fig. 11(c)], indicating that the PIV plane now cuts through the two elliptic points $E$ and $E^{*}$ [see Fig. 12(c)].

${ }^{28}$ E. Stone and P. Holmes, "Random perturbations of heteroclinic attractors," SIAM J. Appl. Math. 50, 726 (1990).

${ }^{29}$ J. Porter and E. Knobloch, "Dynamics in the $1: 2$ spatial resonance with broken reflection symmetry," Physica D 201, 318 (2005). 